\title{
A NONLINEAR APPROACH FOR PREDICTING STOCK RETURNS AND VOLATILITY WITH THE USE OF INVESTOR SENTIMENT INDICES ${ }^{\$}$
}

\author{
Stelios BeKIROS $^{\mathrm{a}, \mathrm{b}}$, RANGAN GUPTA $^{\mathrm{a}, \mathrm{c}}{ }^{* *}$ and CLEMENT KYEI ${ }^{\mathrm{c} * *}$ \\ ${ }^{\mathrm{a}} I P A G$ Business School, ${ }^{\mathrm{b}}$ European University Institute (EUI), ${ }^{\mathrm{c}}$ University of Pretoria
}

\begin{abstract}
The popular sentiment-based investor index $S^{B W}$ introduced by Baker and Wurgler $(2006,2007)$ is shown to have no predictive ability for stock returns. However, and Huang et al. (2015) developed a new investor sentiment index, $S^{P L S}$, which they show can predict monthly stock returns based on a linear framework. However, the linear model may lead to misspecification and lack of robustness. We provide statistical evidence that the relationship between stock returns, $S^{B W}$ and $S^{P L S}$ is characterized by structural instability and inherent nonlinearity. Given this, using a nonparametric causality approach, we show that neither $S^{B W}$ or $S^{P L S}$ predicts stock market returns or even its volatility, as opposed to previous empirical evidence.
\end{abstract}

JEL Codes: C22, C32, C53, G10, G11

Keywords: Investor sentiment; stock markets; nonlinear dependence

\section{INTRODUCTION}

As pointed out by Huang et al. (2015), investor sentiment can affect asset prices as market agents tend to make overly optimistic or pessimistic judgments and choices. However, trader behaviour and investor sentiment is not directly measurable or observable. Based on that fact, Baker and Wurgler (2006, 2007; hereafter BW) constructed a novel sentiment index, which aggregates information from six proxies namely close-end fund discount rates, share turnover, number of initial public offerings (IPOs), first-day returns of IPOs, dividend premium and equity share in new issues. The Baker and Wurgler $(2006,2007)$ index is created via a principal components methodology. Recently, Huang et al. (2015) using the same

\footnotetext{
${ }^{\$}$ We would like to thank two anonymous referees for many helpful comments. However, any remaining errors are solely ours.

* Corresponding author: ${ }^{a}$ IPAG Business School, 184 Boulevard Saint-Germain, 75006 Paris, France.; Tel.: +33 0153633600 ; Fax: +33014544 $4046 ;{ }^{b}$ Department of Economics, Via della Piazzuola; 43, I-50133, Florence, Italy; Tel.: +39 0554685916 ; Fax: +39 055 4685 902; E-mail address: stelios.bekiros@eui.eu.

${ }^{* * *}{ }^{c}$ Department of Economics, University of Pretoria, Pretoria, 0002, South Africa. E-mail address: rangan.gupta@up.ac.za.

${ }^{* * * *}{ }^{c}$ Department of Economics, University of Pretoria, Pretoria, 0002, South Africa. E-mail address: kweku.shaker@gmail.com.
} 
proxies of BW developed a new "aligned investor sentiment index", which separates out the information in the proxies that is relevant to the expected stock returns from the error or noise, utilizing a partial least squares (PLS) method. Importantly, Huang et al. (2015) showed that their modified index can predict aggregate stock market returns at a monthly frequency, as opposed to the results by Baker and Wurgler (2007) and Baker et al. (2012).

Note that, in their pioneering study, Baker and Wurgler (2006) use the first principal component (PC) of the proxies as the measure of investor sentiment. From the point of view of econometrics, the first principal component is the best combination of the six proxies that maximally represents the total variations of theese six proxies. However, all the proxies may have approximation errors to the true but unobservable investor sentiment, and these errors too form parts of their variations. Given this, the first principal component can potentially contain a substantial amount of common approximation errors that are not relevant for predicting stock returns. Against this backdrop, the objective of Huang et al. (2015) is to align the investor sentiment measure with the purpose of explaining the stock returns by extracting the most relevant common component from the six proxies. One one hand, from point of view of economics, Huang et al. (2015) separate out information in the proxies that is relevant to the expected stock returns from the error or noise. On the other hand, from the perspective of statistics, the PLS method, developed by Wold $(1966,1975)$ and later extended by Kelly and Pruitt $(2013,2015)$, achieves exactly this objective. This new index extracted using PLS is what Huang et al. (2015) calls the aligned investor sentiment index that incorporates efficiently all the relevant forecasting information from the six proxies, which is proven by these authors to be the case using forecast encompassing tests in their applications.

The objective of our work is to compare the predictive ability of the Baker and Wurgler (2006, 2007) sentiment index $\left(S^{B W}\right)$ against that of Huang et al. (2015) (denoted as $S^{P L S}$ ) not only for the aggregate stock market returns, but also for their volatility, using the nonparametric causality test of Nishiyama et al. (2011). This test is developed to incorporate higher-order interrelationships inherently 
based on the nonlinear dependence structure between the investigated variables in question. Our assessment to use a nonparametric approach also stems from the fact that the behaviour of stock returns and volatility vis-à-vis $S^{B W}$ and $S^{P L S}$ might be highly nonlinear.

It is worth noting that Huang et al. (2015) obtained their results on the predictability of stock returns via the $S^{P L S}$ index, based on a linear regression framework. Whilst this is undoubtedly most widely used in the literature (e.g., Rapach and Zhou, 2013), yet if statistical testing reveals nonlinearity the existence of which we demonstrate thereafter - the results are unreliable. This is the first paper, to the best of our knowledge, which utilizes a nonlinear nonparametric framework to compare the forecastability of $S^{B W}$ and $S^{P L S}$ vis-à-vis market returns and their volatility, the latter of which has never been examined whatsoever. The rest of the paper is organized as follows: Section 2 presents the empirical methodology, while Section 3 discusses the data and presents the results. Finally, Section 4 concludes.

\section{Methodology}

The methodology by Nishiyama et al. (2011) explores high-order causalities assuming the following nonlinear dependence between the investigated series:

$$
x_{t}=g\left(x_{t-1}\right)+\sigma\left(y_{t-1}\right) \epsilon_{t}
$$

where $\left\{x_{t}\right\}$ and $\left\{y_{t}\right\}$ are stationary processes and $g($.$) and \sigma($.$) are unknown functions which satisfy$ certain conditions for stationary. In general, $y_{t-1}$ carries information in predicting $x_{t}^{K}$ for a given $K$, hence the null hypothesis of non-causality in the $K^{\text {th }}$ moment is given by:

$$
H_{0}: E\left(x_{t}^{K} \mid x_{t-1}, \ldots, x_{1}, y_{t-1}, \ldots, y_{1}\right)=E\left(x_{t}^{K} \mid x_{t-1}, \ldots, x_{1}\right) \text { w.p. } 1 \text {. }
$$

where w.p. 1 abbreviates to "with probability one". Formally, $y_{t}$ does not cause $x_{t}$ up to the $K^{\text {th }}$ moment if:

$$
H_{0}: E\left(x_{t}^{K} \mid x_{t-1}, \ldots, x_{1}, y_{t-1}, \ldots, y_{1}\right)=E\left(x_{t}^{K} \mid x_{t-1}, \ldots, x_{1}\right) w . p .1 \text {. for all } k=1, \ldots, K
$$


Nishiyama et al. (2011) in their work provide a detailed description of how to construct the test statistic $\hat{S}_{t}^{(k)}$ for any $k=1, \ldots, K$. We implement the test for $k=1$ to test for non-causality in the $1^{\text {st }}$ moment (mean) and for $k=2$ in the $2^{\text {nd }}$ moment, i.e., non-causality in variance.

\section{EMPIRICAL ANALYSIS}

The aggregate stock market returns are estimated as the excess returns of a market index, which is common in the relevant literature. Specifically we calculate the continuously compounded log-returns of the S\&P 500 index (including dividends) minus the risk-free rate. The return (spr) of the S\&P 500 and its volatility $(s p v)$ measured as the squared values of the returns, are derived from the Center for Research in Security Prices (CRSP). The data on both the value-adjusted CSRP for the S\&P500 index and the risk free rate can be found at http://www.hec.unil.ch/agoyal/, while the sentiment indexes $S^{B W}$ and $S^{P L S}$ can be downloaded from http://apps.olin.wustl.edu/faculty/zhou/Sentiment_BW_PLS.txt, already standardized. Our monthly sample covers the period 1965:7 - 2010:12.

Initially we employ the standard linear Granger causality test for complementarity reasons. To ensure that our results will be comparable vs. the nonparametric test, we use a lag-length of one in the vector autoregressive (VAR) modelling. Note that for the case of $s r$ and $S^{B W}$, the Schwarz Information Criterion (SIC) chooses a lag-length of one, but it chooses a lag-length of eight for the VAR comprising of $s r$ and $S^{P L S}{ }^{1}$ We decided to use a lag-length of one for the sake of comparability across the two investor sentiment indices. Also, this is in line with the predictive regression framework of Huang et al., (2015), which is essentially a one-lag-based VAR. Further, as indicated above, recall that the nonparametric test is designed to use one lag only. As is well-known, higher lag orders leads to

\footnotetext{
${ }^{1}$ We also experimented with alternative lag-length selection criteria (with maximum lags set at twenty-four), namely, the Likelihood Ratio (LR), Akaike Information Criterion (AIC), Final Prediction Error (FPE) and Hannan-Quinn (HQ). When we used a lag-length of eight, we found that $S^{P L S}$ no longer caused $s r$ at the 5 percent level of significance. For the VAR comprising of $s r$ and $S^{P L S}$, the LR, FPE and AIC suggested nineteen lags, while the HQ suggested fourteen lags. Whether we used nineteen or fourteen lags, we still could not reject the null that SPLS does not Granger cause sr at the 5 percent level of significance. For the case of $S^{B W}$ and $s r$, the LR, AIC, and FPE suggested thirteen lags, while the HQ, as with the SIC, suggested one lag. Interestingly at thirteen lags, we rejected the null that $S^{B W}$ does not Granger cause $s r$ at the one percent level of significance. In any event, the results for the linear model cannot be relied upon in the presence of nonlinearity and regime changes. Complete details of these results are available upon request from the authors.
} 
overparametrization problems in nonparametric approaches. As it is demonstrated in Table 1, the null hypothesis that $S^{B W}$ and $S^{P L S}$ does not Granger cause $s p r$ can be strongly rejected only for the latter case, thus confirming the results of Huang et al. (2015).

\section{TABle 1: Linear Granger-Causality TeST}

Dependent Variable: $\operatorname{spr}(1965: 7-2010: 12)$
$S^{B W}$
1.4043
$S^{P L S}$
$8.6395 * * *$

Note: $* * *$ indicates rejection of the null hypothesis of absence of Granger causality at $1 \%$ level.

Next, we conduct the Bai and Perron (2003) test for detecting multiple structural breaks in case of an autoregressive AR(1) model for $s p r$, as well as for a bivariate VAR(1) including $S^{B W}$ or $S^{P L S}$ respectively. We were not able to detect any structural breaks for the AR(1) model, yet interestingly five breaks were obtained by the VAR model between $s p r$ and $S^{B W}$ (i.e., 1970:7, 1974:10, 1977:1, 2000:9 and 2008:9), and between $s p r$ and $S^{P L S}$ (i.e., 1972:6, 1974:10, 1987:10, 1991:3 and 2008:10). In the presence of these breaks, the assumption of parameter constancy over the entire sample as inferred by the linear Granger causality test is strongly violated, and consequently cannot be deemed conclusive. It must be noted that the break dates obtained under the VARs comprising of $s p r$ and $S^{B W}$, and $s p r$ and $S^{P L S}$ are in general quite different except for the break at 1974:10. It is difficult to precisely explain why this is the case, but it can be speculated that this is most likely due to the fact that two investor sentiments, though comprising of the same underlying variables, are generated by different approaches, as discussed in the introduction.

Furthermore, we use the Brock et al. (1996) test for the non-iid null hypothesis on the residuals of the spr AR(1) model and of the VAR comprising spr and $S^{B W}$ or $S^{P L S}$ respectively. It is illustrated from Table 2, that the test overwhelmingly rejects the null of iid structure for many embedding dimensions, thus implying an omitted nonlinear structure. In order to substantiate the evidence of structural breakpoints, nonstationarity and possible nonlinear interdependencies, and eventually deal 
TABLE 2: BDS TEST

\begin{tabular}{cccc}
\hline Dimension & $\operatorname{AR}(1)$ & $S^{B W}$ - based VAR(1) & $S^{P L S}$ - based VAR(1) \\
\hline 2 & 0.0049 & 0.0055 & 0.0095 \\
3 & 0.0007 & 0.0006 & 0.0009 \\
4 & 0.0000 & 0.0000 & 0.0000 \\
5 & 0.0000 & 0.0000 & 0.0000 \\
6 & 0.0000 & 0.0000 & 0.0000 \\
\hline \hline
\end{tabular}

Note: Entries are $p$-values for the null of serial independence in the error structure of $s p r$ after using an AR(1) filter or two VAR(1) model specifications i.e., $\left[\mathrm{spr}, S^{B W}\right]$ and $\left[\mathrm{spr}, S^{P L S}\right]$ respectively

with the misspecification of our linear modelling, we utilize the nonparametric causality test proposed by Nishiyama et al. (2011). The results are displayed in Table 3. Surprisingly now, there is no evidence of even $S^{P L S}$ acting as a predictor for $s p r$ now. As with the linear Granger causality test, $S^{B W}$ is still not found to cause spr. These results also carry over to the volatility series (spv) as well. Note that, even though there is no statistical evidence of predictability, the nonparametric testing still tends to suggest that $S^{P L S}$ is a stronger predictor compared to $S^{B W}$ both for returns and volatility. Based on the findings of nonlinear structural relationships between stock returns and the two investor sentiment indices, we deem the detected causality results for $S^{B W}$ and $S^{P L S}$ obtained by the Nishiyama et al., (2011) test, as far more robust and reliable when compared to the linear granger causality test.

\section{TABle 3: NONPARAMETRIC CAUSALITY TEST}

Dependent Variable: $s p r$ and $s p v(1965: 7-2010: 12)$

\begin{tabular}{ccc}
\hline \hline & \multicolumn{2}{c}{ Test statistics } \\
\cline { 2 - 3 }$S^{B W}$ & $\hat{S}_{T}^{(1)}$ & $\hat{S}_{T}^{(2)}$ \\
$S^{P L S}$ & 1.7184 & 9.1030 \\
& 3.4857 & 12.3548 \\
\hline
\end{tabular}

Note: $5 \%$ critical values is $14.38 ; \hat{S}_{T}^{(1)}$ : Test statistic for causality in-mean; $\hat{S}_{T}^{(2)}$ : Test statistic for causality in-variance 


\section{CONCLusions}

As market sentiment is not directly observable, Baker and Wurgler $(2006,2007)$ created a novel investor index $\left(S^{B W}\right)$ that aggregates behavioural information from six financial proxy indicators using principal components analysis. More recently, Huang et al. (2015) using the same proxies developed a new index $\left(S^{P L S}\right)$ which distinguishes information from the observed expected stock returns vis-à-vis error or noise signals in the market, via a partial least squares approach. Based on a linear predictive regression model, they showed that their modified index could predict aggregate stock market returns at monthly frequencies.

In the present work, we showed that due to inherent nonlinearities in the interrelationship between returns and the two sentiment indexes, the linear Granger causality framework upon which Huang et al. (2015) relied, might lead to misspecification. In this regard, we employed the nonparametric causality test of Nishiyama et al. (2011) to demonstrate that, in fact, just like the $S^{B W}$ index, the $S^{P L S}$ index too fails to predict not only stock returns but also its volatility. In future research, it would be interesting to use the powerful frequency domain-based Granger causality test of Breitung and Candelon (2006) to verify our results from the linear Granger causality test. This test allows us to detect causality at short-, medium, and longer-horizons. However note, in the presence of nonlinearity and structural breaks, which we show exists in the data and in the relationships between stock returns and the investor sentiment indexes, the Breitung and Candelon (2006) test, which too is based on a linear framework, will also be misspecified as well. In addition, it would be interesting to verify whether our results are robust to out-of-sample forecasting or not, using various nonlinear models.

\section{REFERENCES}

Bai, J. and Perron, P. 2003. Computation and analysis of multiple structural change models. Journal of Applied Econometrics 18, 1-22.

Baker, M., and Wurgler, J. 2006. Investor sentiment and the cross-section of stock returns. Journal of Finance 61, 1645-1680. 
Baker, M., and Wurgler, J. 2007. Investor sentiment in the stock market. Journal of Economic Perspectives 21, 129-152.

Baker, M., Wurgler, J., and Yuan, Y. 2012. Global, local, and contagious investor sentiment. Journal of Financial Economics 104, 272-287.

Breitung, J., and Candelon, B. (2006). Testing for short and long-run causality: A frequency domain approach. Journal of Econometrics 132, 363-378.

Brock, W., Dechert, D., Scheinkman, J. and LeBaron, B. (1996). A test for independence based on the correlation dimension. Econometric Reviews 15, 197-235.

Huang, D., Jiang, F., Tu, J., and Zhou, G. 2015. Investor sentiment aligned: a powerful predictor of stock returns. Review of Financial Studies 28, 791-837.

Kelly, B., and S. Pruitt. 2013. Market expectations in the cross-section of present values. Journal of Finance 68: 1721-1756.

Kelly, B., and S. Pruitt. 2015. The three-pass regression filter: A new approach to forecasting using many predictors. Journal of Econometrics, 186(2): 294-316.

Nishiyama, Y., Hitomi, K., Kawasak, Y. and Jeong, K. 2011. A consistent nonparametric test for nonlinear causality - Specification in time series regression. Journal of Econometrics 165, 112-127.

Rapach and Zhou 2013. Forecasting Stock Returns, David E. Rapach and Guofu Zhou, in Handbook of Economic Forecasting, Volume 2A, Graham Elliott and Allan Timmermann (Eds.), Amsterdam: Elsevier (September 2013), 328-383.

Wold, H. 1966. Estimation of principal components and related models by iterative least squares. In P. R. Krishnaiaah (eds.), Multivariate Analysis, 391-420. New York: Academic Press.

Wold, H. 1975. Path models with latent variables: The NIPALS approach. In H. M. Blalock, A. Aganbegian, F. M. Borodkin, R. Boudon, and V. Cappecchi (eds.), Quantitative Sociology: International Perspectives on Mathematical and Statistical Modeling. New York: Academic Press. 